\title{
Breath sound analyses of infants with respiratory syncytial virus-induced acute bronchiolitis
}

\author{
Kenta Kuruma ${ }^{1}$, Tomofumi Otomo ${ }^{1}$, Takashi Sakama ${ }^{1}$, Kousuke Akiyama ${ }^{1}$, Hiromitsu \\ Takakura $^{1}$, Daisuke Toyama ${ }^{1}$, Kota Hirai ${ }^{1}$, Hiroyuki Furuya ${ }^{2}$, Masahiko Kato ${ }^{3}$, and \\ Hiroyuki Mochizuki² \\ ${ }^{1}$ Tokai University Hachioji Hospital \\ ${ }^{2}$ Tokai University School of Medicine \\ ${ }^{3}$ Tokai Daigaku Igakubu
}

January 23, 2022

\begin{abstract}
Introduction: The reliability of breath sound analysis using an improved method in infants has been reported. Objective: Breath sounds of infants with respiratory syncytial virus (RSV)-induced acute bronchiolitis were analyzed to evaluate their specificity and examine their relationship with the severity. Subjects and methods: We evaluated the inspiratory and expiratory breath sound parameters of 33 infants (median age $=2$ months old) diagnosed with RSV-induced acute bronchiolitis. The sound powers of inspiration and expiration and the spectrum curve indices (ratio of the third and fourth area to the total area under the curve and ratio of the power and frequency at $50 \%$ and $75 \%$ of the highest frequency were evaluated at the acute phase and recovery phase. Furthermore, the relationship between the breath sound parameters and the clinical severity of acute bronchiolitis was examined. Results: Analyses of the breath sound spectrogram showed that the power of expiration at the acute phase was large, and was the expiration-to-inspiration sound power ratio in a mid-frequency range (E/I MF), with values decreased in the recovery phase. Data of the inspiratory sound spectrum curve showed the improvement of airway stenosis during the recovery period. Furthermore, there were significant relationships between the E/I MF and SpO2 and the severity score of acute bronchiolitis. Conclusion: In RSV-induced acute bronchiolitis, specificity of breath sounds was observed in the expiration sound and the reversible airway stenosis was evaluated. Breath sound analyses are expected to be useful for the diagnosis and clinical evaluation of the severity of acute bronchiolitis.
\end{abstract}

\section{ORIGINAL ARTICLE}

\section{Article title}

Breath sound analyses of infants with respiratory syncytial virus-induced acute bronchiolitis

\section{Author Listing}

Kenta Kuruma, M.D. ${ }^{1,2}$, Tomofumi Otomo, M.D. ${ }^{1,3}$,

Takashi Sakama, M.D. ${ }^{1,3}$, Kosuke Akiyama, M.D., Ph.D. ${ }^{1,3}$,

Hiromitsu Takakura, M.D. ${ }^{1,3}$, Daisuke Toyama, M.D., Ph.D. ${ }^{1,3}$,

Kota Hirai, M.D., Ph.D. ${ }^{1,3}$, Hiroyuki Furuya, M.D., Ph.D. ${ }^{4}$,

Masahiko Kato, M.D., Ph.D. ${ }^{1,3}$, Hiroyuki Mochizuki, M.D., Ph.D. ${ }^{1,3}$

Author Affiliation 
1; Department of Pediatrics, Tokai University Hachioji Hospital, ${ }^{2 ;}$ Tokyo metropolitan children's medical center, ${ }^{3 ;}$ Department of Pediatrics, Tokai University School of Medicine, ${ }^{4}$; Department of Basic Clinical Science and Public Health, Tokai University School of Medicine

\section{Corresponding Author}

Hiroyuki Mochizuki, M.D., Ph.D.

Department of Pediatrics, Tokai University Hachioji Hospital

Ishikawa-cho 1838, Hachioji, Tokyo, JAPAN, Post code: 192-0032

Phone: +81-42-639-1111, Fax: +81-42-639-1112

E-mail: mochihi@tokai-u.jp

Short Title: Breath sound analyses of acute bronchiolitis

Key words: bronchiolitis, breath sound analysis, respiratory syncytial virus

Funding Information

This study was supported by the Environmental Restoration and Conservation Agency of Japan in fiscal years 2019-2021. No.1-1.

\section{Abbreviations}

RSV: respiratory syncytial virus

E LF: the expiration sound power in a low-frequency range

I LF: the inspiration sound power in a low-frequency range

E MF: the expiration sound power in a mid-frequency range

I MF: the inspiration sound power in a mid-frequency range

$\mathrm{E} / \mathrm{I} \mathrm{LF}$ : the expiration-to-inspiration sound power ratio in a low-frequency range

E/I MF : the expiration-to-inspiration sound power ratio in a mid-frequency range

AUC: area under the curve

$\mathrm{A}_{\mathrm{T}}$ : total area under the curve of $100 \mathrm{~Hz}$ to the highest frequency of the $\mathrm{dBm}$ power spectrum

$\mathrm{A}_{3}$ : third area under the curve

$\mathrm{B}_{4}$ : fourth area under the curve

$\mathrm{RPF}_{50}$ : ratio of power and frequency at $50 \%$ of the highest frequency of the $\mathrm{dBm}$ power spectrum

$\mathrm{RPF}_{75}$ : ratio of power and frequency at $75 \%$ of the highest frequency of the $\mathrm{dBm}$ power spectrum

\section{Abstract}

Introduction: The reliability of breath sound analysis using an improved method in infants has been reported.

Objective: Breath sounds of infants with respiratory syncytial virus (RSV)-induced acute bronchiolitis were analyzed to evaluate their specificity and examine their relationship with the severity.

Subjects and methods: We evaluated the inspiratory and expiratory breath sound parameters of 33 infants (median age $=2$ months old) diagnosed with RSV-induced acute bronchiolitis. The sound powers of inspiration and expiration and the spectrum curve indices (ratio of the third and fourth area to the total area under the curve and ratio of the power and frequency at $50 \%$ and $75 \%$ of the highest frequency were 
evaluated at the acute phase and recovery phase. Furthermore, the relationship between the breath sound parameters and the clinical severity of acute bronchiolitis was examined.

Results: Analyses of the breath sound spectrogram showed that the power of expiration at the acute phase was large, and was the expiration-to-inspiration sound power ratio in a mid-frequency range (E/I MF), with values decreased in the recovery phase. Data of the inspiratory sound spectrum curve showed the improvement of airway stenosis during the recovery period. Furthermore, there were significant relationships between the $\mathrm{E} / \mathrm{I} \mathrm{MF}$ and $\mathrm{SpO}_{2}$ and the severity score of acute bronchiolitis.

Conclusion: In RSV-induced acute bronchiolitis, specificity of breath sounds was observed in the expiration sound and the reversible airway stenosis was evaluated. Breath sound analyses are expected to be useful for the diagnosis and clinical evaluation of the severity of acute bronchiolitis.

\section{Introduction}

During the COVID-19 epidemic, Japan experienced an unprecedented outbreak of respiratory syncytial virus (RSV) in the summer of 2021 like other nations ${ }^{1)}$, and many infants who visited hospitals were diagnosed with acute bronchiolitis. Acute bronchiolitis caused by RSV is a common disease in infants, but some cases are turned severe $^{2)}$. Furthermore, infants who have the RSV-induced acute bronchiolitis suffer from subsequent episodes of recurrent wheezes at a constant rate ${ }^{3)}$. According to the recent reports, RSV-induced acute bronchiolitis is considered to be a trigger for the onset of childhood asthma ${ }^{4}$, and there is some concern that it may have a significant impact on the future respiratory function of patients ${ }^{5}$.

Of note, no specific treatment for acute bronchiolitis has been established. Although the efficacy of inhaled $\beta_{2}$ agonists, adrenaline and hypertonic saline and systemic steroids continues to be discussed ${ }^{6,7)}$, no satisfactory conclusions with clinical efficacy have yet been obtained. One serious problem concerning therapy for acute bronchiolitis is the difficulty of assessing the severity of acute bronchiolitis in the acute phase ${ }^{8)}$, which is related to the difficulty in performing objective lung function tests, such as a spirogram, due to patient tolerance issues, as acute bronchiolitis typically has an onset under two years of age ${ }^{9)}$.

Recently, the utility of a breath sound analysis as a simple, safe and non-invasive lung function test has been studied, and technological progress in this field has been remarkable ${ }^{10,11)}$. Clinically, current reports have suggested that new breath sound analyses can be used in the clinical evaluation of airway changes ${ }^{12)}$, and have evaluated the lung function using breath sound analyses in children ${ }^{13)}$. The possibility to objectively evaluate the airway condition in infants has also been reported ${ }^{14,15)}$. In the present study, we have examined the characteristics of breath sounds in infants under two years old with RSV-induced acute bronchiolitis and investigated the relationship between various breath sound parameters and the severity scores and clinical symptoms in these patients.

\section{Subject and methods}

\section{1, Subjects}

From June 1 to August 31, 2021, a retrospective observational study was conducted on 55 infants (male: female $=27: 28$, median age $=5$ months old), who were admitted to our hospital for respiratory infection caused by RSV, and underwent a routine breath sound analysis ${ }^{13,16)}$. In all patients, the clinical course, severity of illness and medical history were checked.

The study protocol was approved by the institutional review board of Tokai University Hospital (IRB number; 21R126, approval date; August 27, 2021), and the opt-outs were conducted at our outpatient clinic (from August 28 to November 30, 2021). For infants who were finally diagnosed with RSV-induced acute bronchiolitis, we informed their legal guardians about the study by letter, and their written informed consent was obtained.

\section{2, Study protocol}

Breath sounds were measured at the time of hospitalization (acute phase) and after discharge (recovery 
phase). The contents of the study were as follows: (1) to examine the specificity of RSV-induced acute bronchiolitis from the breath sound spectrogram, (2) to examine the power of expiratory and inspiratory sounds ${ }^{17,18)}$ and to compare them between the acute phase and the recovery phase, (3) to compare the various parameters of sound spectrum curve indices between the acute phase and the recovery phase, and (4) to evaluate the relationship between the various breath sound parameters and the $\mathrm{SpO}_{2}$ values, the severity score of acute bronchiolitis ${ }^{19)}$ and the number of days of hospitalization.

The diagnosis of acute bronchiolitis was made by more than two specialists with pediatric licenses. They diagnosed acute bronchiolitis according to previous reports ${ }^{8,20,21)}$, infants younger than two years, who had respiratory symptoms and wheezes and/or rhonchi and had decreased $\mathrm{SpO}_{2}$ and hyperinflation findings on chest radiographs.

The clinical severity of acute bronchiolitis was based on the clinical score of De Boeck, et al. ${ }^{19)}$. Their score was determined based on the $\mathrm{SpO}_{2}$ value, respiratory rate, presence of expiratory wheezes/rhonchi and chest retraction. Each of these four items was divided into four stages from none, mild, moderate and severe, and assigned 0 to 3 points. We evaluated the total points of this score in each patient.

All patients received equal treatment with oxygen administration, humidification, removal of airway secretions and administration of expectorants. Inhalation of $\beta_{2}$-agonists and hypertonic saline and treatment with systemic steroids were not performed. No patients needed mechanical ventilation.

\section{3, Breath sound analysis method}

Breath sound analyses were performed for all participants, as described previously ${ }^{13,16)}$. Breath sounds were recorded using a handheld microphone for [?]10 seconds. The microphone was placed on the right upper anterior chest at the second intercostal space along the mid clavicular line. A sound analysis of the inspiration phase was performed using an LSA-2012 sound spectrometer (Kenz Medico Co., Saitama, Japan).

The sound-amplifying unit was found to be effective for analyzing sounds in the range of 100-3,000 Hz. The recorded sounds were analyzed according to the fast Fourier transformation. The sampling frequency was $10,240 \mathrm{~Hz}$ and the spectra were obtained using a Hamming window. The sounds were displayed as a sound spectrogram. The point of the maximum frequency $(\mathrm{Hz})$ in the shape during inspiration was used for the sound spectrum analysis.

To evaluate the dBm-based spectrum images, we used a new analyzing program (LSA 2020/ANA, Kenz Medico Co., Saitama, Japan). Using this program, we decided to set the zero point of the Y-axis (dBm) based on the mean of the background noise of all subjects. In this report, the zero level $(0 \mathrm{~dB}$ of breath sound spectrum) was visually corrected based on the breath sound spectrums in each sample before the zero point (the frequency at $0 \mathrm{~dB}$ ) was decided ${ }^{22)}$. The zero level and the zero point were used to calculate of the area under the curve (AUC) of the sound spectrum.

From the breath sound spectrum, the power of low frequencies (LF) (100-200 Hz) and middle frequencies (MF) (200-400 Hz) in the inspiration and the expiration sounds were calculated ${ }^{17)}$. In addition, the inspiration-to-expiration sound power ratio in the low- and mid-frequency ranges (E/I LF and E/I MF, respectively) were also calculated ${ }^{17)}$.

The sound spectrum curve indices of the inspiration were determined by one point of the maximum frequency $(\mathrm{Hz})$ in the sound spectrum. The data were automatically calculated using a custom software program ${ }^{22}$. The spectrum curve indices $\left(\mathrm{A}_{3} / \mathrm{A}_{\mathrm{T}} \mathrm{B}_{4} / \mathrm{A}_{\mathrm{T}}, \mathrm{RPF}_{75}\right.$ and $\left.\mathrm{RPF}_{50}\right)$ were measured according to the methods of a previous report ${ }^{13,16)}$. The $\mathrm{RPF}_{75}$ is the ratio of power at $\mathrm{F}_{75}\left(\mathrm{dBF}_{75}\right)$ to the frequency value $\left(\mathrm{F}_{99}-\mathrm{F}_{75}\right)$, and the $\mathrm{RPF}_{50}$ is the ratio of power at $\mathrm{F}_{50}\left(\mathrm{dBF}_{50}\right)$ to the frequency value $\left(\mathrm{F}_{99}-\mathrm{F}_{50}\right)$. The total sound spectrum was divided into three or four sections from low to high frequencies, which allowed for the quantitation of the energy distribution within the spectrum $\left.{ }^{13}, 16\right)$. The $A_{T}, A_{3}$ and $B_{4}$ were conventionally calculated by $\mathrm{dBm}$ and $\mathrm{Hz}$ (1 arbitrary unit $[\mathrm{dBmHz}]$ on a spectrum image. The $\mathrm{A}_{3} / \mathrm{A}_{\mathrm{T}}$ and $\mathrm{B}_{4} / \mathrm{A}_{\mathrm{T}}$ values are the ratio of the higher-frequency areas $\left(\mathrm{A}_{3}\right.$ and $\left.\mathrm{B}_{4}\right)$ to the total area $\left(\mathrm{A}_{T}\right)$ of the sound spectrum ${ }^{13,16)}$. 
A five-point moving average was used as a smoothing technique to determine the suitable $\mathrm{dB}$ value for identifying the $0 \mathrm{~dB}$ in the slope of each sound spectrum. Each personal breath sound parameter was analyzed conventionally, using a sample with a median value from three tidal breaths.

\section{4, Statistical analyses}

The statistical analyses were conducted using the SPSS software program (IBM SPSS Statistics, Version 22 for Windows; IBM Corp., Armonk, N.Y., USA). The parameters were compared using Wilcoxon's signed-rank test. $P$ values of $<0.05$ were considered to indicate statistical significance. Bonferroni's multiple comparison test was used for multiple comparisons, and in Table 2, $P$ values of $<0.017$ were considered to indicate statistical significance among the power of I, E and E/I. Table data are mainly expressed as the median and the first and the third quartile values.

\section{Results}

\section{1, Subjects profile}

Of the 55 patients hospitalized with RSV-induced respiratory tract infections, 38 were diagnosed with acute bronchiolitis at less than two years of age (male: female $=18: 20$, median age $=3$ months old). All patients were negative for COVID-19 and had no history of recurrent wheezing and asthma. After discharge, 5 of the 38 patients were not able to revisit our hospital due to family reasons. The comparison of data between the acute phase and the recovery phase was thus performed for 33 subjects (males: females $=14$ : 19, median age $=2$ months old)(Figure 1 ).

A history of food allergy was present in $3(9.1 \%)$ and atopic dermatitis was reported in $2(6.1 \%)$. On admission, a fever was present in 24 patients (72.7\%) and cough in 31 patients (93.9\%) (Table 1). Chest X-ray revealed pneumonia in 8 patients $(24.2 \%)$ of the patients. Chest auscultation by a pediatric specialists showed wheezes and/or rhonchi in all patients. Chest radiographs showed some degree of evidence of hyperinflation in both lungs in all patients.

The median time between the first and second measurements of breath sounds was 10 days. Chest auscultation by the pediatric specialists revealed no respiratory adventitious sounds in any patients at the outpatient booth.

\section{2, The Evaluation of the breath sound spectrogram}

On comparing the respiratory sounds of patients in the recovery period and a seven-month-old healthy infant from a previous study ${ }^{15)}$ to those of patientsint the acute phase of acute bronchiolitis, the sound power of inspiration and expiration in the acute phase were great, especially that of expiration (Figure 1).

An examination of the breath sound spectrogram images of all the patients showed sinusoidal waveforms of wheezes $(>400 \mathrm{~Hz},>200 \mathrm{mS})$ in $11(33 \%)$, sinusoidal waveforms of rhonchi ([?]200 Hz, >200mS) in $7(21$ $\%$ ), and non-uniform, granular bands suggesting strong power in the low-pitched region of the expiratory spectrum (Figure 1) in 20 (61\%, 5 cases overlapped with wheezes or rhonchi). As a typical case of this sound, the sound spectrum on expiration in one patient showed a waveform with several peaks (Figure 2).

\section{3, Results of breath sound analyses}

The sound power of respiration was large, and the E/I LF and E/I ML were also large at the acute phase, significantly decreasing in the recovery phase (Table 2 ).

All sound spectrum curve indices of inspiration, including $A_{3} / A_{T}, B_{4} / A_{T}, R P F_{50}$ and $R P F_{75}$, were significantly increased in the recovery phase (Table 2).

\section{4, Breath sound parameters and clinical severity}

There was a significant correlation between the log. E/I MF and the oxygen saturation on admission $(\mathrm{p}=0.010)$, and between the E/I MF and the severity score of acute bronchiolitis $(\mathrm{p}=0.004)$ (Table 3$)$. There was no correlation with the number of hospitalization days. 


\section{Discussion}

Recent advances in breath sound analyses have been remarkable ${ }^{10,11)}$. In clinical practice, attempts have been made to use breath sound analyses for the obstructive evaluation of airway narrowing ${ }^{23,24)}$, and the technique is improving ${ }^{18,22)}$. Since the measurement of breath sounds is non-invasive, simple and can be obtained with effortless breath, studies are underway concerning its application for the evaluation of respiratory diseases in children ${ }^{16)}$ and infants ${ }^{14)}$ who cannot perform normal lung function tests.

In the present study, of note, the breath sound spectrogram of expiration in acute bronchiolitis was found to be characteristic. As previously mentioned ${ }^{25,26)}$, the breath sounds of acute phase of acute bronchiolitis show marked power that is not observed in the breath sound spectrogram of healthy children ${ }^{27)}$ or children with mild exacerbation of asthma ${ }^{16)}$. According to reports of the pathophysiology ${ }^{28)}$, epithelial damage and desquamation that introduce clusters of desquamated epithelial cells and viscous sputum ${ }^{29}$ ) are severe in the patients with acute bronchiolitis. Thus, a remarkable increase in the expiratory sound power of infants with acute bronchiolitis may be induced by severe stenosis of airways associated with these causes.

In this report, attending physicians described the presence of rhonchi, which are low-pitched continuousexpiratory sounds ${ }^{30)}$, as a respiratory adventitious sound during expiration in many patients detected on auscultation. Widely, acute bronchiolitis is known to cause wheezes ${ }^{2)}$, which are high-pitched continuousexpiratory sounds ${ }^{30)}$, but our data included fewer patients with wheezes than previous reports on breath sounds $\left.{ }^{25}, 26\right)$. Typical rhonchi and wheezes are continuous musical sounds ${ }^{31}$ ) that are easy to identify as a sinusoidal waveform in the breath sound spectrogram ${ }^{27}$. However, based on examination of breath sound spectrograms, the expiratory sounds may differ from the waveform sounds of rhonchi and wheezes which commonly found in asthmatic children. We suspect that the non-uniform, band-like sounds in the lowpitched area seen in Figure 1 were heard as rhonchi. The sound spectrum of the expiration showed several repetitive peaks (Figure 2), which we presumed to be a pulse train or "complex repetitive waveforms", as suggested by Tal, et al. ${ }^{25)}$. We will continue to investigate the cause of such characteristic sound in infants with acute bronchiolitis.

Due to the increased power of the expiration, the E/I values were extremely large, showing a significant decrease in the recovery period. The $\mathrm{E} / \mathrm{I} \mathrm{MF}$ values in the recovery period were almost the same as those in healthy infants (the median value of $\mathrm{E} / \mathrm{I} \mathrm{MF}$ in 26 healthy 1-month-old infants who visited our hospital $=0.05$, private data). The airway stenosis in the acute bronchiolitis ${ }^{28,29)}$ may be stronger than that in the acute exacerbation of asthma ${ }^{32)}$, although these results cannot be simply compared, as the airway portion with stenosis may differ between these conditions, and the subjects with acute bronchiolitis were younger than asthmatic children on average ${ }^{9,33)}$.

Despite these uncertainties, we speculate that our outstanding finding will prove useful for the diagnosis of acute bronchiolitis in infants. Although this study was a single-institutional, short-term study, we were able to limit the target to patients with RSV-induced acute bronchiolitis within the same season in an environment where pediatric respiratory tract infections are almost nonexistent due to the COVID-19 epidemic. Acute bronchiolitis should produce special sounds different from bronchoconstriction induced by smooth muscle constriction $^{33)}$, which may be particularly meaningful in distinguishing this condition from infantile asthma.

According to previous reports of adults, the $\mathrm{E} / \mathrm{I}$ LF value in healthy adults is 0.36 , which is equivalent to that in infants, although the $\mathrm{E} / \mathrm{I} \mathrm{MF}$ value is large at $0.27^{17)}$. This difference was perplexing, as the I MF value of infants $\left(87.2 \mathrm{~dB}\right.$ ) was larger than that of adults $\left.(57.3 \mathrm{~dB})^{17}\right)$. The cause of this result is speculated to be dependent on the difference in airway diameter, respiratory flow rate (L/sec) and respiration pattern between adults and infants.

The values of the four sound spectrum curve indices in the inspiratory sounds ${ }^{13,16)}\left(\mathrm{A}_{3} / \mathrm{A}_{\mathrm{T}}, \mathrm{B}_{4} / \mathrm{A}_{\mathrm{T}}, \mathrm{RPF}_{75}\right.$ and $\mathrm{RPF}_{50}$ ) being significantly increased during the recovery period is attributed to the presence of bronchial constriction during the acute phase, based on previous breath sound analysis results ${ }^{15}$. In the present study, respiratory symptoms have improved in all patients at the second visit, and there were no adventitious sounds with auscultation. Thus, the changes in breath sounds during the recovery period are considered to indicate 
direct improvement of the airways.

More interestingly, a significant correlation was found between the E/I MF and $\mathrm{SpO}_{2}$, and even the severity score of bronchiolitis ${ }^{19}$. Although no correlation was found with the length of hospital stay, the correlation with the severity score used in clinical practice seems to be helpful for creating more objective severity scores. A number of studies have reported that RSV-induced acute bronchiolitis was associated with recurrent wheezing in later childhood ${ }^{3)}$ and that the rate of development of asthma was not small ${ }^{4}$. Our results seems to be important for establishing more precise severity scores with an objective markers.

One limitation of our report is that we were unable to clearly demonstrate the specificity of the acute bronchiolitis due to the lack of data on the healthy age-matched infants (1-3 months old). Thus, we hope to explore this a topic in the future. Furthermore, we were not able to indicate whether or not the same findings would be obtained in other cases of infantile wheezing diseases with dyspnea ${ }^{33)}$. However, this problem is not easily resolved, as the infants with acute bronchiolitis are mainly $<6$ months old ${ }^{9}$, and asthma, which is a representative of childhood wheezing diseases with dyspnea, cannot be diagnosed at this period ${ }^{33,34)}$. In a study of older children with asthma, it was reported that an intentional loud breathing increases the power of respiration ${ }^{35)}$. There is no doubt that the breathing at rest in infants with acute bronchiolitis is greater than that in normal infants, so the specificity of breath sounds of acute bronchiolitis must be evaluated under such situation. However, the apparent significant increase in the expiration power is very clear, and we hope to evaluate this issue in the future.

Many discussions have been held concerning the effective treatment of acute bronchiolitis. According to the previous reports, inhaled $\beta_{2}$ agonists have been shown to be ineffective, while inhaled adrenaline, inhaled saline, systemic steroids and their combination have been reported to be effective ${ }^{5-7)}$. We hope to revisit our evaluation of the advantages of a breath sound analysis ${ }^{13,16)}$. Objective data over time using breath sound analyses may facilitate a clear evaluation of the effect of pharmacotherapy. As there are no clinical objective lung function tests available for infants ${ }^{33}$, 34), our findings are considered clinically meaningful. We are now considering a method to objectively evaluate the effects of drugs using a breath sound analysis.

Our results confirmed the specificity of the breath sounds in RSV-induced acute bronchiolitis. It has been reported that the breath sound directly reflects the condition of airways ${ }^{13,16)}$. Our results seem to be useful for differentiating the diagnosis of acute bronchiolitis from other wheezing diseases, creating a more accurate severity scores and determining the objective effect of treatment.

\section{Author Contributions}

Kenta Kuruma: Conceptualization (equal), data curation (equal), investigation (equal), project administration (equal), visualization (equal), writing original draft (equal), writing review and editing (equal), Tomofumi Otomo: Data curation (equal), investigation (equal), writing original draft (equal), Takashi Sakama: Data curation (equal), investigation (equal), writing original draft (equal),Kosuke Akiyama: Data curation (equal), investigation (equal), writing original draft (equal), Hiromitsu Takakura: Data curation (equal), investigation (equal), writing original draft (equal),Daisuke Toyama: Data curation (equal), investigation (equal), methodology (equal), supervision (equal), writing original draft (equal), Kota Hirai: Conceptualization (equal), data curation (equal), investigation (equal), methodology (equal), supervision (equal), writing original draft (equal), writing review and editing (equal), Hiroyuki Furuya: Methodology (equal), formal analysis (equal), validation (equal), software (equal), supervision (equal), writing review and editing (equal), Masahiko Kato:Conceptualization (equal), methodology (equal), validation (equal), formal analysis (equal), supervision (equal), resources (equal), writing review and editing (equal), Hiroyuki Mochizuki:Conceptualization (equal), methodology (equal), formal analysis (equal), supervision (equal), project administration (equal), validation (equal), resources (equal), writing original draft (equal), writing review and editing (equal),

\section{Acknowledgement}

We would like to thank Dr. Terufumi Shimoda Department of Allergy, San Remo Rehabilitation Hospital, 
for his kind guidance in calculating of expiration and inspiration power.

\section{Potential Conflict of Interest}

The authors have no potential conflicts of interest to disclosure.

\section{ORCID}

Hiroyuki Mochizuki: https://orcid.org/0000-0002-5273-1820

Kenta Kuruma: https://orcid.org/0000-0003-2198-2018

\section{References}

1. Agha R, Avner JR. Delayed Seasonal RSV Surge Observed During the COVID-19 Pandemic. Pediatrics . 2021; 148 : e2021052089.

2. Shi T, McAllister DA, O'Brien KL, Simoes EAF, Madhi SA, Gessner BD, Polack FP, Balsells E, Acacio S, Aguayo C, et al. RSV Global Epidemiology Network. Global, regional, and national disease burden estimates of acute lower respiratory infections due to respiratory syncytial virus in young children in 2015: a systematic review and modelling study. Lancet. 2017; 390 : 946-958.

3. Mochizuki H, Kusuda S, Okada K, Yoshihara S, Furuya H, Simões EAF. Palivizumab prophylaxis in preterm infants and subsequent recurrent wheezing. Six-year follow-up study. Am J Respir Crit Care Med . 2017; 196 : 29-38.

4. Sigurs N, Bjarnason R, Sigurbergsson F, Kjellman B. Respiratory syncytial virus bronchiolitis in infancy is an important risk factor for asthma and allergy at age 7. Am J Respir Crit Care Med . 2000; 161 : 1501-1507.

5. Backman K, Piippo-Savolainen E, Ollikainen H, Koskela H, Korppi M. Irreversible airway obstruction in adulthood after bronchiolitis in infancy: evidence from a 30-year follow-up study. Respir Med . 2014; $108: 218-223$.

6. Plint AC, Johnson DW, Patel H, Wiebe N, Correll R, Brant R, Mitton C, Gouin S, Bhatt M, Joubert G, et al. Pediatric Emergency Research Canada (PERC). Epinephrine and dexamethasone in children with bronchiolitis. N Engl J Med . 2009; 360 : 2079-2089.

7. Zhang L, Mendoza-Sassi RA, Wainwright C, Klassen TP. Nebulised hypertonic saline solution for acute bronchiolitis in infants. Cochrane Database Syst Rev . 2017; 12 : CD006458.

8. Ralston SL, Lieberthal AS, Meissner HC, Alverson BK, Baley JE, Gadomski AM, Johnson DW, Light MJ, Maraqa NF, Mendonce EA, et al. Clinical practice guideline: the diagnosis, management, and prevention of bronchiolitis. Pediatrics . 2014; 134 : e1474-502.

9. Hall CB, Weinberg GA, Iwane MK, Blumkin AK, Edwards KM, Staat MA, Auinger P, Griffin MR, Poehling KA, Erdman D, et al. The burden of respiratory syncytial virus infection in young children. $N$ Engl J Med. 2009; 360 : 588-598.

10. Grzywalski T, Piecuch M, Szajek M, Breborowicz A, Hafke-Dys H, Kociński J, Pastusiak A, Belluzzo R. Practical implementation of artificial intelligence algorithms in pulmonary auscultation examination. Eur J Pediatr 2019; 178 : 883-890.

11. Palnitkar H, Henry BM, Dai Z, Peng Y, Mansy HA, Sandler RH, Balk RA, Royston T. Sound transmission in human thorax through airway insonification: an experimental and computational study with diagnostic applications. J Med Biol Eng Comput. 2020;58 : 2239-2258.

12. Oweis RJ, Abdulhay EW, Khayal A, Awad A. An alternative respiratory sounds classification system utilizing artificial neural networks.Biomed J. 2015; 38 : 153-161.

13. Tabata H, Hirayama M, Enseki M, Nukaga M, Hirai K, Furuya H, Mochizuki H. A novel method for detecting airway narrowing using breath sound spectrum analysis in children. Respir Invest , 2016;54 : 20-28.

14. Enseki M, Nukaga M, Tabata H, Hirai K, Matsuda S, Mochizuki H. A clinical method for detecting bronchial reversibility using a breath sound spectrum analysis in infants. Respir Invest, 2017;55 : 219-228. 
15. Shioya H, Tadaki H, Yamazaki F, Miyamoto M, Yoshihara S, Enseki M, Tabata H, Hirai K, Furuya $\mathrm{H}$, Kato M, et al. Characteristics of breath sound in infants with risk factors for asthma development.Allergol Int . 2019; 68 : 90-95.

16. Nukaga M, Tabata H, Enseki M, Hirai K, Furuya H, Kato M, Mochizuki H. Changes in the breath sound spectrum with bronchodilation in children with asthma. Respir Investig . 2018; 56 : 392-398.

17. Shimoda T, Obase Y, Nagasaka Y, Nakano H, Kishikawa R, Iwanaga T. Lung sound analysis and airway Inflammation in bronchial asthma. J Allergy Clin Immunol Pract . 2016; 4 : 505-511.

18. Shimoda T, Obase Y, Nagasaka Y, Nakano H, Kishikawa R, Iwanaga T. Airway inflammation phenotype prediction in asthma patients using lung sound analysis with fractional exhaled nitric oxide. Allergol Int . 2017; 66 : 581-585.

19. De Boeck K, Van der Aa N, Van Lierde S, Corbeel L, Eeckels R. Respiratory syncytial virus bronchiolitis: a double-blind dexamethasone efficacy study. J Pediatr , 1997; 131 : 919-921.

20. Friedman JN, Rieder MJ, Walton JM. Bronchiolitis: recommendations for diagnosis, monitoring and management of children one to 24 months of age. Paediatr Child Health , 2014; 19 : 485-498.

21. Dawson KP, Long A, Kennedy J, Mogridge N. The chest radiograph in acute bronchiolitis. J Paediatr Child Health. 1990;26 : 209-211.

22. Koike H, Imamura T, Murayama Y, Tabata H, Enseki M, Furuya H, Niimura F, Mochizuki H. New technique to analyze the breath sound spectrum in children with asthma. Tokai J Exp Clin Med . 2020; 45 : 195-201.

23. Pasterkamp H, Kraman SS, Wodicka GR. Respiratory sounds, Am J Respir Crit Care Med. 1977; 156 : 974-987.

24. Malmberg LP, Pesu L, Sovijarvi ARA. Significant differences in flow standardized breath sound spectra in patients with chronic obstructive pulmonary disease, stable asthma, and healthy lungs. Thorax 1995;50 : 1285-1291.

25. Tal A, Sanchez I, Pasterkamp H. Respirosonography in infants with acute bronchiolitis. Am J Dis Child . 1991; 145 : 1405-1410.

26. Takase M. The unique features in the lung sounds of acute bronchiolitis. Clinical Pharmacology and Therapy . 2010;20 : 25-28. (Japanese).

27. Murayama Y, Shioya H, Tadaki H, Miyamoto M, Yoshihara S, Tabata H, Furuya H, Uchiyama A, Mochizuki H. Objective evaluation of wheezing in normal infants. Pediatr Int. 2019; 61 : 956-961.

28. Folkerts G, Nijkamp FP. Virus-induced airway hyperresponsiveness. Role of inflammatory cells and mediators. Am J Respir Crit Care Med . 1995; 151 : 1666-1673.

29. Yamada Y, Yoshihara S. Creola bodies in infancy with respiratory syncytial virus bronchiolitis predict the development of asthma. Allergol Int . 2010; 59 : 375-380.

30. Pasterkamp H, Kraman SS, Wodicka GR. Respiratory sounds. Advances beyond the stethoscope. Am J Respir Crit Care Med . 1997;156 : 974-987.

31. Gavriely N, Palti Y, Alroy G, Grotberg JB. Measurement and theory of wheezing breath sound. $J$ Appl Physiol. 1984; 57 : 481-492.

32. Imamura T, Enseki M, Furuya H, Niimura F, Mochizuki H. Changes in the Breath Sound Spectrum with Bronchodilator Inhalation in Asthmatic Children with Long-term Management. Tokai J Exp Clin Med. 2020;45 : 24-30.

33. Global Initiative for Asthma. Global Strategy for Asthma Management and Prevention. http://ginasthma.org/wp-content/uploads/2021/05/GINA-Main-Report-2021. Accessed:12 January, 2022.

34. Mochizuki H, Hirai K, Tabata H. Forced oscillation technique and childhood asthma. Allergol Int . 2012; 61 : 373-383.

35. Kagawa T, Imamura T, Enseki M, Tabata H, Furuya H, Niimura F, Mochizuki H. Effect of inspiratory flow on breath sound analysis in children with asthma. Arerugi , 2020; 69 : 184-191 (Japanese).

\section{Table and Figure Legends}

Table 1. Characteristics of the infants diagnosed with RSV-induced acute bronchiolitis 
a: median (first quartile, third quartile), b: from Reference No. 19.

Table 2. Results of the analyses of parameters of the breath sound spectrum of infants diagnosed with RSV-induced acute bronchiolitis

a: median (first quartile, third quartile), b: Wilcoxon's signed-rank test. Bold letters represent values with a significant difference.

Table 3. Relationship between parameters of the breath sound spectrum and clinical factors of infants diagnosed with RSV-induced acute bronchiolitis

a: Pearson's correlation coefficient. Bold letters represent values with a significant difference.

Figure 1. A sound spectrogram of a patient with RSV-induced acute bronchiolitis and normal infant

Spectrograms of a patient with RSV-induced acute bronchiolitis; the acute phase, the recovery phase and a seven-month-old health infant are shown. After a Fourier analysis, the vertical axis showing the frequency in $\mathrm{Hz}$ and the horizontal axis showing time. The sound intensity of the breath sounds is indicated by the color. Ex: expiration, Ins: inspiration.

Figure 2. The expiratory sound spectrum in a patient with RSV-induced acute bronchiolitis

The expiratory sound spectrum in a patient with RSV-induced acute bronchiolitis of Figure 1 shows several distinct speaks, similar to a previous report (reference No. 26).

Table 1. Characteristics of the infants diagnosed with RSV-induced acute bronchiolitis

Number Age Sex Complication Length of Severity of

of (Months) (m:f) Fever Cough Pneumonia Hospitalization Bronchiolitis ${ }^{b}$

Subjects (\%) (\%) (\%) (Days)

$332^{\mathrm{a}} 14: 192436861$

$(1,10)(72.7)(93.9)(24.2)(4,8)(1,3)$

a: Median (first quartile, third quartile), b: Reference No. 19.

Table 2. Results of analyses of parameters of the breath sound spectrum in infants diagnosed with RSVinduced acute bronchiolitis

Acute Phase Recovery Phase P-value ${ }^{b}$

【Expiration and Inspiration Sound Power】

Power of I LF (dB) $96.4(87.5,109.6)^{\mathrm{a}} 96.4(87.5,109.6) 0.097$

Power of E LF (dB) $96.9(87.1,113.6) 81.6(74.9,85.8)$

$<0.001$

E/I LF $0.93(0.44,3.52) 0.30(0.11,0.77)<\mathbf{0 . 0 0 1}$

Power of I MF (dB) $92.1(83.1,103.2) 87.2(80.3,95.1) \mathbf{0 . 0 1 5}$

Power of E MF (dB) $78.9(63.0,104.3) 58.3(50.3,66.0)$

$<0.001$

E/I MF $0.27(0.06,0.97) 0.04(0.02,0.07)<\mathbf{0 . 0 0 1}$

【Inspiration spectrum Curve Indices】

$\mathrm{A}_{3} / \mathrm{A}_{\mathrm{T}}(\%) 17.0(14.6,19.9) \quad 19.0(17.2,20.9) \mathbf{0 . 0 1 1}$ 
$\mathrm{B}_{4} / \mathrm{A}_{\mathrm{T}}(\%) 10.4(9.6,12.6) 12.2(11.1,14.1) \mathbf{0 . 0 0 3}$

$\mathrm{RPF}_{75} 7.1(5.7,8.3) \quad 8.3(7.2,10.2) \mathbf{0 . 0 0 1}$

$\mathrm{RPF}_{50} 5.4(4.7,6.0) \quad 5.9(5.5,7.1) \mathbf{0 . 0 4 6}$

a: Median (first quartile, third quartile), b: Wilcoxon's signed-rank test. Bold letters represent values with a significant difference.

Table 3. Relationship between parameters of the breath sound spectrum and clinical factors of infants diagnosed with RSV-induced acute bronchiolitis

Oxygen Severity of Length of Saturation Bronchiolitis Hospitalization

CC p-value ${ }^{\mathrm{a}} \mathrm{CC}$ p-value CC p-value

【Expiration and Inspiration Sound Power】

Power of I LF (dB) -0.062 $0.730-0.0640 .7250 .0610 .737$

Power of E LF (dB) -0.134 0.456 0.089 0.620 0.126 0.486

Power of I ML (dB) - 0.2020 .2590 .0150 .9350 .1220 .498

Power of E ML (dB) -0.133 0.461 0.080 0.658 0.0930 .609

LogE/I LF -0.111 0.538 0.164 0.3610 .1010 .575

LogE/I MF - $0.440 \quad \mathbf{0 . 0 1 0} 0.487 \mathbf{0 . 0 0 4} 0.0620 .733$

【Inspiration spectrum Curve Indices】

$\mathrm{A}_{3} / \mathrm{A}_{\mathrm{T}}-0.1110 .537-0.0750 .6800 .0810 .656$

$\mathrm{B}_{4} / \mathrm{A}_{\mathrm{T}}-0.1520 .398-0.0390 .8310 .0220 .904$

$\mathrm{RPF}_{75}-0.2300 .1980 .0690 .7010 .1190 .511$

$\mathrm{RPF}_{50} 0.1390 .441-0.0380 .8330 .0040 .981$

CC: correlation coefficient, a: Pearson's correlation coefficient. Bold letters represent values with a significant difference.

Figure 1

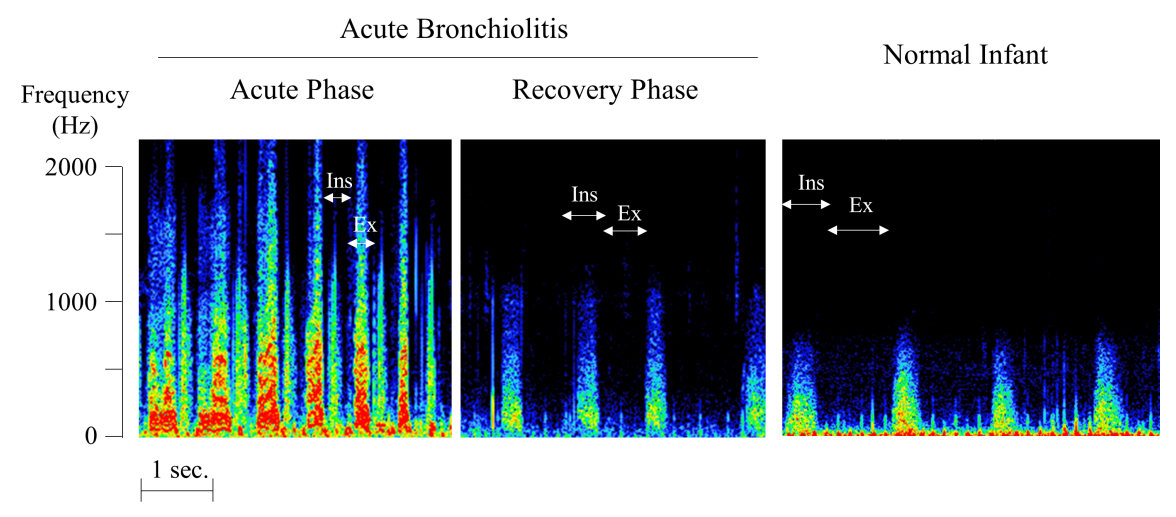


Figure 2

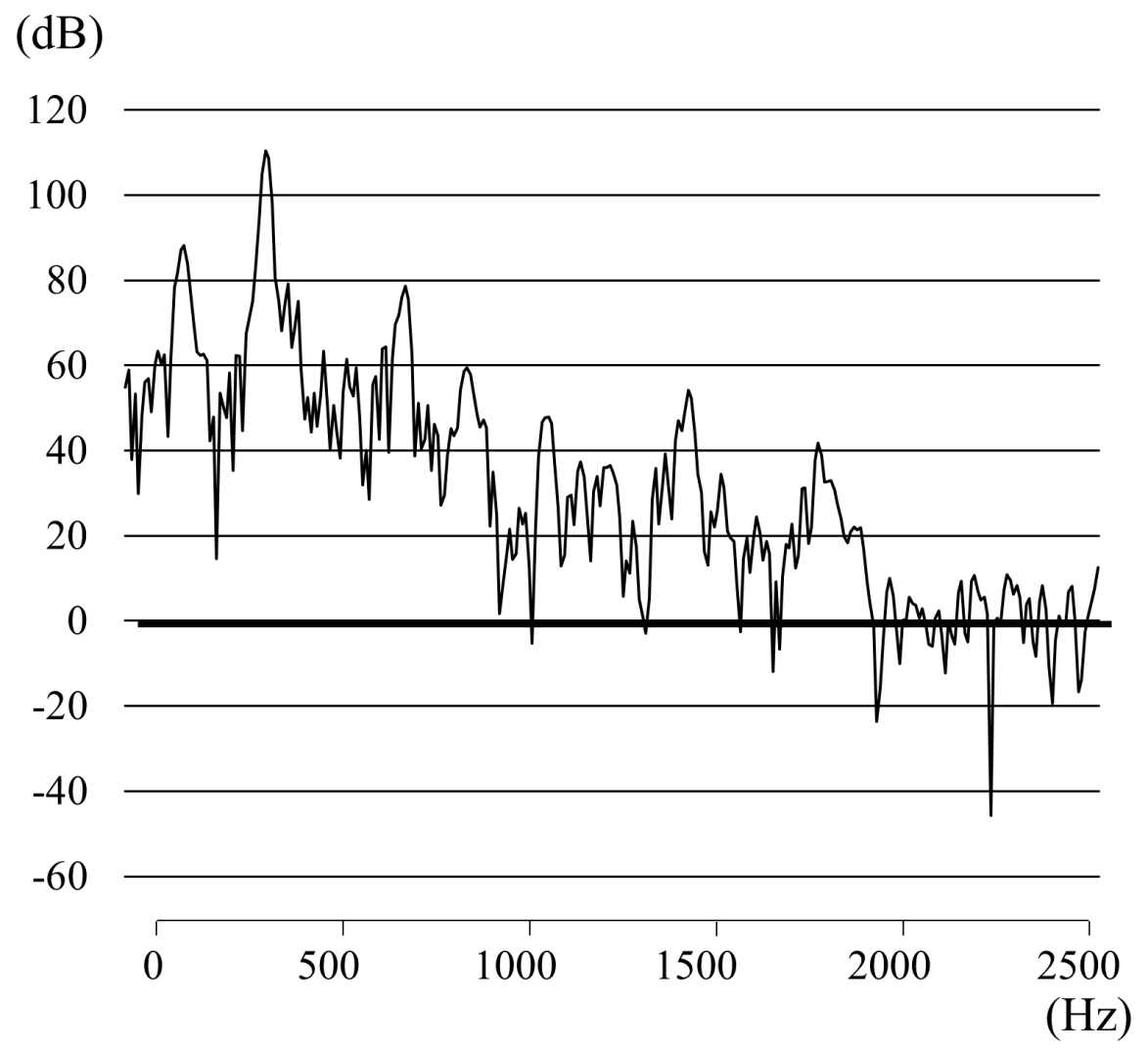

